\title{
Chronic Analgesic (NSAID induced) SJS: A Case Report
}

\author{
Dave Riya $^{1}$, Rajinder Sharma ${ }^{1}$, Kuber Sharma ${ }^{1}$, Lakshya Yadav ${ }^{1}$ \\ Resident, Department of General Medicine, SGT Medical College, Gurugram
}

\begin{abstract}
In the contemporary era, use of drugs is the dominant paradigm of health care. The most quotidian drug used for fever and pain is paracetamol. Although adverse reactions to paracetamol in India are rare, at times they can cause life-threatening situations. Stevens-Johnson syndrome (SJS) is one such potentially lethal adverse drug reaction. The most reported cases of analgesic-induced SJS were due to oxicams or propionic acid derivatives. There are very few detailed reports of SJS due to the use of paracetamol. We report a case of SJS, which occurred due to the use of paracetamol. The clinical features of this condition and multidisciplinary management of the patient are described in brief.
\end{abstract}

Keywords: Adverse drug reaction, paracetamol, Stevens-Johnson syndrome

\section{Introduction}

Steven-Johnson syndrome (SJS) is an infrequent and a severe form of erythema multiforme (EM). It can occur due to an adverse hypersensitivity reaction to drugs which results in skin and mucosal eruptions that can be potentially fatal. It is considered to be a less severe form of toxic epidermal necrolysis (TEN). The only difference being the extent of epidermal detachment; that is, $30 \%$ of the total body surface area; while $10-30 \%$ is known as SJS-TEN overlap ${ }^{[1]}$

"A new eruptive fever with stomatitis and ophthalmia" was described as a severe variant of EM and was termed by Steven and Johnson in 1922.[2]

SJS may present as a nonspecific febrile illness leading to malaise, headache, cough, rhinorrhea with polymorphic lesions of the skin and mucous membrane characterized by acute blisters and erosions.

\section{Corresponding author:}

Dr. Lakshya Yadav

Resident, Department of General Medicine

SGt Medical College

lakshyayadav@gmail.com,9728626052
The incidence of SJS has been estimated to be around 1-6/1,000,000 persons per year with a mortality rate of $1-5 \%$ which rises up to $30 \%$ in TEN. Multiple drugs have been identified to cause SJS and TEN, antibiotics (sulfonamides) being the most common $^{[3]}$

The drugs that cause SJS commonly are antibacterials (sulfonamides), anticonvulsants (phenytoin, phenobarbital, and carbamazepine), nonsteroidal anti-inflammatory drugs (oxicam derivatives), and oxide inhibitors (allopurinol).[4]

Paracetamol is among the most extensively used analgesic and anti-pyretic because of easy availability and cost-effectiveness. Despite being considered relatively safe, adverse reactions including cutaneous hypersensitivity reactions have been reported ${ }^{[5]}$

\section{Case Report :}

A 67-year-old male patient reported to Department of Medicine with a chief complaint of painful ulceration of lips and oral cavity leading to difficulty in opening mouth and eating food since 5 days. History of present illness revealed that there was 
burning sensation followed by ulcers which appeared first in oral cavity, lips, and other parts of body including chest, arms, legs and thighs, and genital organs . The redness of eye was evident, and there was a history of watery discharge. Numerous healed lesions were also seen on the chest, axilla which gave the typical appearance of "target lesions" or "bull's eye "appearance".

The past medical history revealed that the patient was suffering from fever and pain since 2 weeks. The patient was prescribed tablet crocin for fever and pain for 7 days by a local medical practitioner. The patient was relieved from fever and pain but later he had burning sensation followed by ulcers in the oral cavity and extra-oral surface.

Intra-oral examination revealed a solitary mixed red and white lesion present on both right and left buccal mucosa. On left buccal mucosa it measured 10 $\mathrm{mm} \times 8 \mathrm{~mm}$ in diameter and right side about $16 \mathrm{~mm} \times$ $9 \mathrm{~mm}$ approximately.

The lesions have well-defined borders and on palpation it was non scrapable and nontender with a rough texture. The upper and lower lips were swollen, and hemorrhagic crusts were present along with profuse bleeding. Laboratory investigations revealed leukocytosis (white blood cells, 15101/1, reference value, 4000-11,000/1) and raised C-reactive protein $59.87 \mathrm{~g} / \mathrm{ml}$; reference range, $0-5 \mathrm{~g} / \mathrm{Ml}$. We had subjected the patient to only the hematological investigation as the lesion being acute, and the patient was under severe discomfort. Based on clinical examination, medical history, and physical examination our diagnosis was SJS.

The patient was treated under an expert guidance of dermatologist with systemic steroids; tablet prednisolone $30 \mathrm{mg}$ twice daily daily for 7 days. Further reduced to $20 \mathrm{mg}$ twice daily for next 7 days. Gradually, $10 \mathrm{mg}$ and $5 \mathrm{mg}$ for consecutive 7 days were administered. Gentian violet application 3 times daily for lips and skin lesions was advocated.

Application of Kenacort (triamcinolone) ointment thrice a day was advised for ulcers in the oral cavity. The patient was reviewed after a week. Lesions had healed significantly in the oral cavity and on the skin. Recall after 2 weeks revealed almost resolved lesions on all the surfaces and completely recovered approximately in 40 days.

\section{Discussion}

SJS is an uncommon, severe, mucocutaneous blistering disorder with an acute and unpredictable onset causing considerable morbidity. Its more severe form is called TEN. Previously, SJS was considered as EM major, but now is considered distinct from EM on the basis of severity, presence of constitutional signs, atypical target lesions with tendency to confluence, positive Nikolsky's sign, more than one mucosal site involvement, and residual sequelae.

In the oral cavity, SJS causes widespread ulcerative lesions. Prodromal symptoms seen in about $30 \%$ of cases and may possibly initiate within 1-3 weeks of starting a new drug, and lasts for 1-2 weeks, presenting with flu-like symptoms, sore throat, headache, arthralgias, myalgias, fever, and other rashes.

Ocular changes such as dry eyes that resemble those of mucous membrane pemphigoid may be noted in few cases. Urethritis and vulval ulcers may occur ${ }^{[6]}$ Our patient did not report any prodrome, but the eye and genital ulcerations were present, along with skin and mouth ulcers.

Although many factors have been proposed as risk factors of SJS, including drug-induced, infections, malignant disorders, and graft rejection, most of them were due to the adverse effect of drugs. The most common drugs are a nonsteroidal 
anti-inflammatory drug (NSAIDs), antipsychotics, antibiotics, allopurinol, and anticonvulsants.

SJS can be differentiated from other skin conditions on three clinical criteria, (i) the pattern of individual skin lesions, (ii) distribution of lesions, and (iii) extent of epidermal detachment.

The characteristic findings in SJS are widespread erythematous or purpuric macules which form flat atypical target lesions as the disease progresses to cause full thickness epithelial necrosis.

Our case showed ulceration of oral cavity, crusting of lips and profuse bleeding, involvement of eye with redness and watery discharge, ulceration of genital region along with numerous healed lesions on chest, axilla which showed typical appearance of "target lesions" or "bull's eye "appearance. The lesions were widespread as compared to EM, which is localized.

\section{Conclusions}

This case report reports the fact that severe hypersensitivity reactions can occur with paracetamol, which can be possibly dangerous and life-threatening. Therefore, clinicians must be more cautious while prescribing. Patients should also be educated regarding the adverse effects of NSAIDs.

Ethical Clearance- Taken from ethical committee of institution

Source of Funding- Self

Conflict of Interest - Nil

\section{References}

1. Khawaja A, Shahab A, Hussain SA. Acetaminophen induced Steven Johnson syndrome-toxic epidermal necrolysis overlap. J Pak Med Assoc. 2012;62:524-7. [PubMed] [Google Scholar]

2. Ramineni HB, Eluri P, Vipparla K, Suryadevara V. Phenobarbital induced Stevens-Johnson syndrome: A case report. Int J Res Med Sci. 2015;3:492-3. [Google Scholar]

3. French LE. Toxic epidermal necrolysis and Stevens Johnson syndrome: Our current understanding. Allergol Int. 2006;55:9-16. [PubMed] [Google Scholar]

4. Deore SS, Dandekar RC, Mahajan AM, Shiledar VV. Drug induced-Stevens Johnson syndrome: A case report. Int J Sci Stud. 2014;2:84-7. [Google Scholar]

5. Kvedariene V, Bencherioua AM, Messaad D, Godard P, Bousquet J, Demoly P. The accuracy of the diagnosis of suspected paracetamol (acetaminophen) hypersensitivity: Results of a single-blinded trial. Clin Exp Allergy. 2002;32:1366-9. [PubMed] [Google Scholar]

6. Patel TK, Barvaliya MJ, Sharma D, Tripathi C. A systematic review of the drug-induced Stevens-Johnson syndrome and toxic epidermal necrolysis in Indian population. Indian $\mathrm{J}$ Dermatol Venereol Leprol. 2013;79:389-98. [PubMed] [Google Scholar] 